\title{
Functions and Relations for a Relativistic Evolving Star with Spherical Symmetry
}

\author{
Ying-Qiu Gu \\ School of Mathematical Science, Fudan University, Shanghai 200433, China \\ Email: yqgu@fudan.edu.cn
}

\begin{abstract}
In this paper, we derive and simplify some important equations and relations for a relativistic evolving star with spherical symmetry, and then give some simple analysis for their properties and implications. In the light-cone coordinate system, these equations and relations have a normal and neat form which is much more accessible than the usual Einstein field equation. The dynamics for the evolving star is reduced to a standard first order hyperbolic partial differential equation system of $(\rho, v)$, which can be analyzed and solved by characteristic method. So they may be helpful to understand the nature of an evolving star and the collapsing process of gravitation.
\end{abstract}

Keywords: stellar structure, stellar evolution, Einstein's field equation, relativistic evolving equation, singularity.

\section{Introduction}

In general relativity, we have some typical exact solutions such as the Schwarzschild, Curzon and Kerr metrics and some of their extensions to the electrovacuum solutions such as the Reissner-Nordström and Kerr-Newman metrics $[1,2,3,4,5,6]$. These solutions shed some lights on the nature of space-time and stars. by

For a static and spherically symmetrical space-time with perfect fluid source, the line element is given

$$
d s^{2}=B(r) d t^{2}-A(r) d r^{2}-r^{2}\left(d \theta^{2}+\sin ^{2} \theta d \varphi^{2}\right),
$$

we have a number of exact solutions $[7,8,9]$ and generating method[10]. In these solutions mass density $\rho$ and pressure $P$ are usually expressed as the functions of $(A, B)$ and their derivatives. In such expressions the properties of the equation of state(EOS) is quite ambiguous, and then most of the solutions are unrealistic in physics.

In fact, the static asymptotically flat space-time with spherical symmetry can be solved by the following procedure. The dynamics for the space-time with perfect fluid can be reduced to the following initial problem of an ordinary differential equation system[11],

$$
\begin{aligned}
M^{\prime}(r) & =4 \pi G \rho r^{2}, & M(0) & =0, \\
\rho^{\prime}(r) & =-\frac{(\rho+P)\left(4 \pi G P r^{3}+M\right)}{C_{s}^{2}(r-2 M) r}, & \rho(0) & =\rho_{0},
\end{aligned}
$$

in which $P=P(\rho)$ is the EOS of the fluid, $M(r)$ is the total mass within the ball of radial coordinate $r$, $C_{s}=\sqrt{P^{\prime}(\rho)}$ is the velocity of sound in the fluid. For any given $\rho_{0}>0$ we get a unique solution. The metric components are given by

$$
A=\left(1-\frac{2 M}{r}\right)^{-1}, \quad B=\exp \left(-\int_{r}^{R} \frac{2\left(4 \pi G P r^{3}+M\right)}{r(r-2 M)} d r\right)
$$

where $R<\infty$ is the radius of the star. $\rho(r)=P(r)=0$ in the region $r \geq R$. 
For any suitable EOS $P=P(\rho)$, the solution of (2) and (3) can be easily solved numerically. By practical calculation, we find that if the EOS satisfying the following increasing and causal conditions

$$
0<C_{s}=\sqrt{\frac{\partial P}{\partial \rho}} \leq \frac{\sqrt{3}}{3}, \quad P \rightarrow\left\{\begin{array}{l}
P_{0} \rho^{\gamma},(\gamma>1, \rho \rightarrow 0), \\
\frac{1}{3} \rho, \quad(\rho \rightarrow \infty),
\end{array}\right.
$$

then the EOS is compatible with space-time and all solutions of (2) and (3) are singularity-free. That is, we always have $0 \leq \rho \leq \rho_{0}$ and $R_{s}=2 M(R)<R$. The condition $\gamma>1$ is necessary, which is caused by inertia of particles and leads to the finite radius of the star $R<\infty$ due to $C_{s} \rightarrow 0$ in (3). The above conclusion can be concretely checked by the following model EOS.

$$
P=\frac{C_{0}^{2} \rho^{1+n}}{k+\rho^{n}}, \quad\left(0<C_{0} \leq \frac{\sqrt{3}}{3}, k>0, n>0\right) .
$$

The star becomes larger as $k \rightarrow 0$ or $n \rightarrow 0$.

However, for an evolutional star, a complete dynamical analysis includes the hydrodynamics of matter, which is too complicated to be solved. In this paper, we consider the simplest case, that is, a star evolves with spherical symmetry. We derive and simplify the dynamical equations in detail, and some simple analysis is given. The results may be helpful to understand the nature of a star and to do further researches.

\section{Dynamics for an Evolving Star}

The line element in the space-time generated by an evolving star with spherical symmetry is generally given by[11]

$$
d s^{2}=u^{2} d \tilde{t}^{2}-(v d \tilde{t}-w d r)^{2}-r^{2}\left(d \theta^{2}+\sin ^{2} \theta d \varphi^{2}\right) .
$$

Here we take the light velocity $c=1$ as unit of speed. For a normal star, $(u, v, w)$ are continuous functions of $(t, r)$ with suitable smoothness. The null geodesic along the radius is described by

$$
(u+v) d \tilde{t}-w d r=0
$$

Assume the solution is $f(\widetilde{t}, r)=C$, where $C$ is a constant. Making light-cone coordinate transformation $t=T(f(\widetilde{t}, r))$, where $T(f)$ is any smooth function satisfying $\partial_{f} T \partial_{\tilde{t}} f>0$, then we get the line element equivalent to $(7)$ as follows $[6,12,13,14,15]$,

$$
d s^{2}=a b d t^{2}+2 \sqrt{b} d t d r-r^{2}\left(d \theta^{2}+\sin ^{2} \theta d \varphi^{2}\right),
$$

where $(a, b)$ are continuous functions of $(t, r)$ with suitable smoothness until the star becomes singular. (8) is similar to the 'Eddington - Finkelstein coordinates'. In this coordinate system, the field equations have very simple form, and some of them are integrable. However, the time coordinate $t$ is different from the usual definition, which should be kept in mind. The usual definition is given by $\delta \tau$,

$$
\delta \tau=\left(\sqrt{a b} d t+\frac{1}{\sqrt{a}} d r\right) c^{-2}
$$

because in this time we have standard form

$$
d s^{2}=c^{2} \delta \tau^{2}-a^{-1} d r^{2}-r^{2}\left(d \theta^{2}+\sin ^{2} \theta d \varphi^{2}\right) .
$$

For the external Schwarzschild solution, in coordinate system (8), we have solution[6]

$$
b=1, \quad a=1-\frac{R_{s}}{r}, \quad\left(\text { for } r \geq R>R_{s}\right),
$$


where $R=R(t)$ and $R_{s}$ are respectively the stellar radius and Schwarzschild radius

$$
R_{s} \equiv 8 \pi G \int_{0}^{R(t)} \rho_{\text {grav }} r^{2} d r
$$

in which $\rho_{\text {grav }}$ is the total gravitational mass-energy density including influences of pressure and momentum. The definition of $\rho_{\text {grav }}$ is given below, and we find $R_{s}$ is a constant.

Denote the 4 -vector speed of the fluid by

$$
U^{\mu}=\{U, V, 0,0\}, \quad U_{\mu}=\{a b U+\sqrt{b} V, \sqrt{b} U, 0,0\},
$$

which satisfies the line element equation

$$
1=g_{\mu \nu} U^{\mu} U^{\nu}=(a b U+2 \sqrt{b} V) U .
$$

For the perfect fluid model, the nonzero components of the energy-momentum tensor $T_{\mu \nu}=(\rho+$ $P) U_{\mu} U_{\nu}-P g_{\mu \nu}$ are given by

$$
\begin{aligned}
& T_{t t}=b(\rho+P)(a \sqrt{b} U+V)^{2}-a b P, \\
& T_{t r}=b(\rho+P)(a \sqrt{b} U+V) U-\sqrt{b} P=T_{r t}, \\
& T_{r r}=b(\rho+P) U^{2}, \quad T_{\theta \theta}=P r^{2}, \quad T_{\varphi \varphi}=P r^{2} \sin ^{2} \theta,
\end{aligned}
$$

where $P=P(\rho)$ is a given EOS which should satisfy increasing and causal conditions (5).

The nonzero components of Einstein tensor are given by

$$
\begin{aligned}
G_{t t} & =-\frac{1}{r}\left(\sqrt{b} \partial_{t} a-a b \partial_{r} a\right)-\frac{1}{r^{2}} a b(1-a), \\
G_{t r} & =\frac{1}{r} \sqrt{b} \partial_{r} a-\frac{1}{r^{2}} \sqrt{b}(1-a)=G_{r t}, \quad G_{r r}=-\frac{\partial_{r} b}{r a}, \\
G_{\theta \theta} & =\left(\frac{a}{r}\left(\frac{\partial_{r} b}{2 b}+\frac{\partial_{r} a}{a}\right)-\frac{1-a}{r^{2}}+\frac{\mathcal{R}}{2}\right) r^{2}, \quad G_{\varphi \varphi}=G_{\theta \theta} \sin ^{2} \theta,
\end{aligned}
$$

where the scalar curvature $\mathcal{R}$ depends on the second order derivatives of the metric functions $(b, a)$. But it is not used in the following discussion, because the related equations are not independent, which can be derived from other equations.

By detailed calculations, we find only the following 3 equations are independent ones in the Einstein equation $G_{\mu \nu}=-8 \pi G T_{\mu \nu}$,

$$
\begin{aligned}
& \partial_{r} b=8 \pi G r(\rho+P) b^{2} U^{2}, \\
& \partial_{t} a=8 \pi G(\rho+P) r V \sqrt{b\left(a+V^{2}\right)}, \\
& \partial_{r} a=-4 \pi G r\left((\rho-P)+(\rho+P) a b U^{2}\right)+\frac{1-a}{r} .
\end{aligned}
$$

By (9), (10) and (12), we learn $\partial_{t} a=0$ if $r>R$, so $R_{s}$ is conserved for an evolving star.

Among the energy-momentum conservation law $T_{; \nu}^{\mu \nu}=0$, only the continuity equation $U_{\mu} T_{; \nu}^{\mu \nu}=0$ is independent, so we get

$$
U^{\mu} \partial_{\mu} \rho+(\rho+P) U_{; \mu}^{\mu}=0 .
$$

Equations (11)-(14) combined with EOS $P=P(\rho)$ form a closed system. The boundary conditions for asymptotic flat space-time are given by

$$
\begin{array}{ll}
\text { at } r=0: \quad a=1, & \partial_{r} a=0, \quad V=0 ; \\
\text { at } r=R: b=1, & a=1-\frac{R_{s}}{R}, \quad \rho=0 .
\end{array}
$$

Together with initial values $\{\rho(0, r), V(0, r)\},(11)$-(16) has a unique solution. 


\section{Simplification of the Equations}

The equations derived above have a weakness, that is, the geometrical variables $(a, b)$ and mechanical variables $(\rho, V)$ couple each other in a complicated manner, which increases the difficulties for discussion. Besides, the physical meaning of $(U, V)$ is unclear, which is quite different from the usual definition $\frac{d r}{d t}$.

To simplify the relations, we introduce the following transformation

$$
U=\sqrt{\frac{1-v}{a b(1+v)}}, \quad V=\frac{\sqrt{a} v}{\sqrt{1-v^{2}}},
$$

where the speed $|v|<1$ is approximately the usual definition. Define an auxiliary energy function by

$$
F \equiv(\rho+P) a b U^{2}=(\rho+P) \frac{1-v}{1+v} .
$$

For a static star, we have $F=\rho+P$. Substituting (17) and (18) into (11)-(13), we get simplified relations

$$
\begin{aligned}
& \partial_{r} a=-8 \pi G r \frac{\rho-P v}{1+v}+\frac{1-a}{r}, \\
& \partial_{r} b=8 \pi G r(\rho+P) \frac{b(1-v)}{a(1+v)}, \\
& \partial_{t} a=8 \pi G r(\rho+P) \frac{a \sqrt{b} v}{1-v^{2}},
\end{aligned}
$$

Obviously, the geometrical variables $(a, b)$ are separated from mechanical ones $(\rho, P, v)$. The solutions can be formally expressed by

$$
\begin{aligned}
& a=1-\frac{8 \pi G}{r} \int_{0}^{r} \frac{\rho-P v}{1+v} r^{2} d r \\
& b=\exp \left(-8 \pi G \int_{r}^{R}(\rho+P) \frac{(1-v) r}{(1+v) a} d r\right),
\end{aligned}
$$

and

$$
a=a(0, r) \exp \left(8 \pi G \int_{0}^{t}(\rho+P) \frac{\sqrt{b} v r}{1-v^{2}} d t\right),
$$

By (13), (18) and (19), for an evolving star, we have

$$
\rho_{\text {grav }}=\frac{1}{2}(\rho-P+F)=\frac{\rho-P v}{1+v} .
$$

For any $\rho(., r) \in L^{\infty}([0, \infty))$, we have $a(\cdot, r) \in C^{0}([0, \infty))$, and it has a positive minimum $a_{\min }>0$. $b(\cdot, r) \in C^{1}([0, R])$ is a monotonic increasing function of $r$. For a normal star, the variables have the following range of value,

$$
0<b \leq 1, \quad 0<a \leq 1, \quad 0 \leq \rho<\infty
$$

Simplifying (14) and the consistent equation of (12) and (13) $\partial_{t r} a=\partial_{r t} a$, we get the dynamical equation for $(\rho, v)$, which is a first order hyperbolic differential equation system,

$$
\begin{aligned}
\left(1-C_{s}^{2}\right) \frac{\partial_{t} \rho}{a \sqrt{b}} & +\left(v+C_{s}^{2}\right) \frac{\partial_{r} \rho}{1-v}+(\rho+P) \frac{\partial_{r} v}{(1-v)^{2}} \\
& =\frac{(\rho+P)}{a(1-v)}\left(4 \pi G r(\rho v-P)-\frac{1}{2 r}[1-a+(1+3 a) v]\right), \\
\left(1-C_{s}^{2}\right) \frac{\partial_{t} v}{a \sqrt{b}} & +(1+v)^{2} C_{s}^{2} \frac{\partial_{r} \rho}{\rho+P}+\left(v+C_{s}^{2}\right) \frac{\partial_{r} v}{1-v} \\
& =\frac{1+v}{a}\left(4 \pi G r\left(C_{s}^{2} \rho v-P\right)-\frac{1}{2 r}\left[1-a+(1+3 a) C_{s}^{2} v\right]\right) .
\end{aligned}
$$


The characteristic speeds are given by

$$
V_{1}=\frac{a \sqrt{b}\left(v+C_{s}\right)}{\left(1-C_{s}\right)(1-v)}, \quad V_{2}=\frac{a \sqrt{b}\left(v-C_{s}\right)}{\left(1+C_{s}\right)(1-v)} .
$$

The disturbance of the solution $(\rho, v)$ propagates at such speed $\frac{d r}{d t}=V_{k}$.

Substituting (19) and (20) into (24) and (25), we get closed equations for $(\rho, v)$, which include all information for an evolving star. Combining (24) and (25) with initial and boundary conditions, we have a unique solution. The numerical solution can be easily solved by method of characteristics.

\section{Analysis for the Solutions}

Since the dynamical equations (24) and (25) are quite complicated, and the rigorous solution is absent. So we can only qualitatively analyze some asymptotic properties of the solutions, and shed some lights on the behavior of an evolving star.

Obviously, when $\rho<\infty$, the star is normal and the space-time should be singularity-free. $\rho \rightarrow \infty$ is a necessary condition for the space-time becoming singular, and $a \rightarrow 0$ or $b \rightarrow 0$ is the signal that the space-time becomes singular.

In (21), $a(0, r)$ is determined by the initial distribution $\rho(0, r)_{\text {grav }}$ via (19), so we have $a>0$. If $\rho \rightarrow \infty$, we have $P \rightarrow C_{0}^{2} \rho$. So according to $(20), b(t, 0) \rightarrow+0$ only if $\rho(t, r) \geq \rho_{0}(t) r^{-2},(r \rightarrow 0)$. This can be checked as follows. For the critical initial distribution

$$
\rho \rightarrow \frac{\rho_{0}}{r^{2}}, \quad P \rightarrow C_{0}^{2} \rho \rightarrow \frac{\rho_{0} C_{0}^{2}}{r^{2}},(r \rightarrow 0) .
$$

When $r \rightarrow+0$, we have $(a \rightarrow 1, v \rightarrow 0)$. For any given $r_{0}$ satisfying $0<r_{0}-r \ll R$, by (20), we have estimation

$$
\begin{aligned}
b(t, r) & =\exp \left(-8 \pi G\left(\int_{r_{0}}^{R}+\int_{r}^{r_{0}}\right)(\rho+P) \frac{(1-v) r}{(1+v) a} d r\right) \\
& \rightarrow A_{1}\left(t, r_{0}\right) \exp \left(-8 \pi G\left(1+C_{0}^{2}\right) \int_{r}^{r_{0}} \frac{\rho_{0}}{r} d r\right) \\
& =A_{2}\left(t, r_{0}\right) r^{8 \pi G \rho_{0}\left(1+C_{0}^{2}\right)} \rightarrow 0, \quad(r \rightarrow 0),
\end{aligned}
$$

in which all $0<A_{k}<\infty$ are independent of $r$. (28) means the space-time itself becomes singular. However, for $\rho(t, r) \rightarrow \rho_{0}(t) r^{-n},(0<n<2)$, we still have $b>0$, and the space-time is still measurable, although the curvature becomes singular.

In the case of a star with extreme high temperature and pressure, the EOS of the fluid becomes simpler. We have the following approximations,

$$
P \doteq C_{0}^{2} \rho, \quad C_{0} \leq \frac{\sqrt{3}}{3} .
$$

Noticing $v(t, 0)=0$, by (19), (20) and (21), near the center $r=0$, we have

$$
|v| \ll 1, \quad b \doteq b_{0}(t), \quad a \doteq 1 .
$$

Substituting (29) and (30) into (24) and (25), we have the simplified dynamical equations which hold near the center of a star,

$$
\begin{aligned}
\partial_{r} w & =\partial_{\eta} w+w\left(1+C_{0}^{-2}\right) \partial_{\eta} v+4 \pi G\left(1+C_{0}^{2}\right) r, \\
\partial_{r} v & =\partial_{\eta} v+w^{-1}\left(1+C_{0}^{2}\right)^{-1} \partial_{\eta} w-\frac{2 v}{r},
\end{aligned}
$$

where $d \eta \equiv \sqrt{b_{0}} d t$ and $w \equiv \rho^{-1}$. Since we have not made approximation for $\rho$, so (31) and (32) are valid for all range value of $\rho$. 
(31) and (32) has a static solution

$$
v=0, \quad \rho=\frac{1}{w}=\frac{1}{C+2 \pi G\left(1+C_{0}^{2}\right) r^{2}},
$$

where $C \geq 0$ is a constant. Even for (31) and (32) with constant characteristic speeds, we can hardly find a rigorous evolving solution. However, (31) is integrable under some ansatz. If we set $v=f(t+r) r$, in this case we have

$$
\frac{1}{\rho}=w=\left(\frac{1}{\rho_{0}(\eta+r)}+\frac{4 \pi G C_{0}^{2}}{f^{\prime}(\eta+r)}\right) \exp \left(-\frac{1+C_{0}^{2}}{2 C_{0}^{2}} \eta(\eta+2 r) f^{\prime}(\eta+r)\right)-\frac{4 \pi G C_{0}^{2}}{f^{\prime}(\eta+r)} .
$$

Of course, under such ansatz, (32) is not strictly satisfied in general. For reasonable function $f(t+r)$, (34) can display local evolving trend of mass density $\rho$ as $(r \rightarrow 0, \eta \rightarrow 0)$.

\section{Discussion and Conclusion}

We derived the dynamics for an evolving star in the light-cone coordinate system. Under some transformation of variables, the equations and relations of dynamics have simple and neat form, which is much more accessible than the usual field equations. The final dynamical equation is reduced to a standard first order hyperbolic system (24) and (25), which can be discussed by method of characteristics.

The singularity analysis for an evolving star is equivalent to discuss the singularity of equation (31) and (32) near the center $r \rightarrow+0$. This is almost a linear first order hyperbolic system with constant characteristic speed. The analysis shows that, it is the EOS of matter and the initial distribution of mass density and speed rather than the total mass-energy to decide the fate of an evolving star.

\section{References}

1. J. N. Islam, Rotating fields in general relativty (Cambridge: Cambridge Univ. Press), 1985.

2. D. Kramer, H. Stephani, E. Herlt, M. MacCallum, E. Schmutzer, Exact Solutions of Einstein's Field Equations, (Cambridge: Cambridge Univ. Press), 1980.

3. J. Bicak, Einstein equations: exact solutions, in Encyclopedia of Mathematical Physics Vol. 2165 (Oxford: Elsevier), 2006.

4. V. S. Manko, J. D. Sanabria-Gómez, O. V. Manko, Nine-parameter electrovac metric involving rational functions, Phys. Rev. D62, 044048(2000).

5. S. Hawking, G. Ellis, The Large Scale Structure of the Space-time, Cambridge Univ. Press 1999.

6. Y. Q. Gu, Exact Vacuum Solutions to the Einstein Equation, Chin. Ann. Math. 28B(5), 2007, 499-506, arXiv:0706.0318

7. M. S. R. Delgaty, K. Lake, Physical Acceptability of Isolated, Static, Spherically Symmetric, Perfect Fluid Solutions of Einstein's Equations, Comput.Phys.Commun.115:395-415,1998, arXiv:gr-qc/9809013

8. K. Lake, All static spherically symmetric perfect fluid solutions of Einstein's Equations, Phys.Rev. D67 (2003) 104015, arXiv:gr-qc/0209104

9. D. Martin, M. Visser, Algorithmic construction of static perfect fluid spheres, Phys.Rev. D69 (2004) 104028, arXiv:gr-qc/0306109

10. P. Boonserm, M. Visser, S. Weinfurtner, Generating perfect fluid spheres in general relativity, Phys.Rev. D71 (2005) 124037, arXiv:gr-qc/0503007

11. S. L. Weinberg, Gravitation and Cosmology, (Ch.5, Ch.11), Wiley, New York, 1972.

12. Y. Q. Gu. Light-Cone Coordiante System in General Relativity, arXiv:0710.5792

13. M. Gasperini, G. Marozzi, F. Nugier and G. Veneziano, Light-cone averaging in cosmology: formalism and applications, JCAP 1107 (2011) 008, [arXiv:1104.1167]

14. F. Scaccabarozzi, J. Yoo, Light-Cone Observables and Gauge-Invariance in the Geodesic Light-Cone Formalism, JCAP 06 (2017) 007, arXiv:1703.08552

15. P. Fleury, F. Nugier, G. Fanizza, Geodesic-light-cone coordinates and the Bianchi I space-time, JCAP 06 (2016) 008, arXiv:1602.04461. 